\title{
Dynamical Properties and Finite-Time Hybrid Projective Synchronization Using Fractional Nonsingular Sliding Mode Surface in Fractional-Order Two-Stage Colpitts Oscillators
}

\author{
Romanic Kengne, ${ }^{1,2}$ Robert Tchitnga, ${ }^{1,2}$ Arnaud Nzeusseu Tchikankou, ${ }^{1,2}$ \\ Aurelle Tchagna Kouanou, ${ }^{1}$ and Anaclet Fomethe ${ }^{3}$ \\ ${ }^{1}$ Laboratory of Electronics and of Signal Processing, Department of Physics, Faculty of Science, University of Dschang, \\ P.O. Box 67, Dschang, Cameroon \\ ${ }^{2}$ Research Group on Experimental and Applied Physics for Sustainable Development (EAPhySuD), P.O. Box 412, Dschang, Cameroon \\ ${ }^{3}$ Laboratoire de Mécanique et de Modélisation des Systèmes, Département de Physique, Faculté des Sciences, Université de Dschang, \\ B.P. 67, Dschang, Cameroon
}

Correspondence should be addressed to Romanic Kengne; kengneromaric@gmail.com

Received 30 August 2013; Accepted 28 October 2013

Academic Editor: Uchechukwu E. Vincent

Copyright (C) 2013 Romanic Kengne et al. This is an open access article distributed under the Creative Commons Attribution License, which permits unrestricted use, distribution, and reproduction in any medium, provided the original work is properly cited.

The dynamics and robust finite-time hybrid projective synchronization of a fractional-order four-dimensional nonlinear system based on a two-stage Colpitts oscillator is investigated. The study of the fractional order stability of the equilibrium states of the system is carried out. The bifurcation diagram confirms the occurrence of Hopf bifurcation in the proposed system when the fractional-order passes a sequence of critical values; the Lyapunov exponent shows the different chaotic sequences of the system. Further, a fractional nonsingular terminal sliding surface and an appropriate robust fractional sliding mode control law are proposed for the finite-time hybrid projective synchronization of a fractional-order chaotic two-stage Colpitts oscillator by taking into account the effects of model uncertainties and the external disturbances. The fractional version of the Lyapunov stability is used to prove the finite-time existence of the sliding motion. Finally, some numerical simulations are presented to demonstrate the effectiveness and applicability of the proposed technique.

\section{Introduction}

Fractional calculus has an about 300-year-old history, but its applications to physics and engineering are rather recent [1]. Many systems are known to display fractional-order dynamics, such as viscoelastic systems, dielectric polarization, and electromagnetic waves [2-4], just to name some.

For some decades, there is a growing interest in investigating the chaotic behavior and dynamics of fractional-order dynamic systems; this can be understood as it has been found that fractional-order systems possess memory and display more sophisticated dynamics compared to their integralorder counterparts, something that is of great significance in secure communication [5-20]. It has been shown that several chaotic systems can remain chaotic when their models become fractional [5]. A three-dimensional fractional-order modified hybrid optical system is presented in [10] where it was shown that Hopf bifurcation occurs on the proposed system when the fractional order varies and passes a sequence of critical values. Despite these many examples the bifurcation of fractional-order nonlinear system has been studied using solely the Caputo derivative definition and limited to threedimensional systems.

On the other hand, in the past two decades, a new direction of chaos research has emerged to address the more challenging problem of chaos synchronization due to its potential applications in laser physics, chemical reactions, secure communication, biomedicine, and so on [21-23]. The thrust of research within this area aims at achieving a master-slave synchronization between two chaotic systems by 
choosing various kinds of methods following the pioneering work of Pecora and Carroll [24]. In [11], based on the stability theory, a novel fractional-order controller is presented for the control and synchronization of the fractional-order Lorenz chaotic system via the fractional derivative. In [12], based on tracking control and the stability theory of nonlinear fractional-order systems, Zhou et al. propose the multidriveone response synchronization technique which is simple and theoretically rigorous. In [13], the hybrid projective synchronization of different dimensional chaotic fractionalorder systems is investigated based on the stability theory of linear fractional-order systems. Based on the fractionalorder stability theory and control tracking, Zhou and Zhu investigate the function projective synchronization for the fractional-order chaotic systems [7]. In [25], based on the stability theory of the fractional-order system, the authors studies on projective synchronization of the new fractionalorder chaotic system through designing the suitable nonlinear controller are investigated. All these examples perfectly clarify the importance of the fractional-order stability theory, but these works have a common drawback from a practical point of view: the lack of knowledge of analytical time of synchronization.

In recent years some researchers have applied finite-time control and synchronization of fractional-order systems by means of the fractional-order Lyapunov stability theory. In [26], the authors design a novel fractional-order nonsingular sliding mode controller for robust synchronization problem of a class of fractional-order chaotic systems in the presence of model uncertainties and external disturbances, the stability of a novel fractional-order integral type sliding surface is proven, based on fractional-order Lyapunov stability theory, a robust sliding control law is derived to guarantee the occurrence of the sliding motion in finite time. In [27], using a nonsingular sliding mode surface, the authors study the finite-time synchronization problem of fractional-order chaotic/hyperchaotic systems in the presence of both model uncertainties and external disturbances. These last works will serve as our handbook in this paper.

It is important to seek bifurcations such as those of Hopf in systems because they are routes towards chaos in such systems. Concerning the system with fractional-order, very little work emphasizes the conditions leading to Hopf bifurcation. Moreover modified projective synchronization is a general case of the simple synchronization. But it had been shown that the finite-time synchronization has very great applications on a practical point of view. Finally, to the best of our knowledge there are few works interested in fractional-order hybrid projective synchronization in finite time and using a fractional nonsingular terminal sliding mode surface; the importance of this surface as that used in [27] is that it supports the robustness of the synchronization in the presence of the disturbances and uncertainties, and that it is easily stabilisable with zero and in finite time.

Motivated by the above discussion, at first in the present work, we propose to tackle the problem of bifurcation of a four-dimensional fractional-order nonlinear system. The two-stage well-studied Colpitts oscillator presented in [28] is a good candidate for the study, due to its broad band in frequency domain. Secondly we study the finite-time hybrid projective synchronization problem of fractional-order twostage Colpitts oscillator in the presence of both model uncertainties and external disturbances. The modified nonsingular terminal sliding mode surface is introduced; its finite-time stability to zero is proved via the Lyapunov stability. So on basis of fractional-order Lyapunov stability theory, a robust control law is designed to force the trajectories of the synchronization error system onto the sliding surface within a finite time and remain on it forever. Numerical simulations demonstrate the applicability and efficiency of the nonlinear control law and verify the theoretical results of the paper.

The rest of this paper is organized as follows. In Section 2, the fractional-order system developed around a two-stage Colpitts oscillator is proposed and its dynamics studied. The numerical results of the dynamics are presented and discussed in Section 3, while the next section is devoted to the synchronization of two two-stage Colpitts oscillators. Finally, Section 5 concludes this work.

\section{The Fractional-Order of a Two-Stage Colpitts Oscillator}

2.1. Dynamics of the System. The proposed four-dimensional fractional-order system under study described by the set of (1) is obtained by modifying the integer-order two-stage Colpitts oscillator proposed in [28]:

$$
\begin{aligned}
& D^{q_{1}} x_{1}=\sigma_{1}\left(x_{4}-\gamma \phi\left(x_{2}+x_{3}\right)\right), \\
& D^{q_{2}} x_{2}=x_{4}, \\
& D^{q_{3}} x_{3}=\sigma_{2}\left(x_{4}-\gamma \phi\left(x_{2}\right)\right), \\
& D^{q_{4}} x_{4}=-x_{1}-x_{2}-x_{3}-\varepsilon x_{4} .
\end{aligned}
$$

Here, the parameters $\sigma_{1}, \sigma_{2}, \gamma$, and $\varepsilon$ are positive reals, $\phi(y)=$ $\exp (-y)-1$, and $q=\left(q_{1}, q_{2}, q_{3}, q_{4}\right)$ is the fractional order. According to [28], when $q=(1,1,1,1)$, the system (1) exhibits chaotic behavior with the parameter values $\sigma_{1}=1.25, \sigma_{2}=1$, $\gamma=1.5385$, and $\varepsilon=1.175$.

\subsubsection{Stability Analysis}

Theorem 1 (see $[15,16])$. The following commensurate order system

$$
{ }_{O}^{C} D_{t}^{q} x(t)=A x(t), \quad x(0)=x_{0},
$$

with $0<q \leq 1, x \in \mathfrak{M}^{n}$, and $A \in \mathfrak{M}^{n \times n}$, is asymptotically stable if and only if $|\arg (\lambda)|>q(\pi / 2)$ is satisfied for all eigenvalues $\lambda$ of the matrix A. Moreover, this system is stable if and only if $|\arg (\lambda)| \geq q(\pi / 2)$ is satisfied for all eigenvalues $\lambda$ of A with those critical eigenvalues that satisfy $|\arg (\lambda)|=q(\pi / 2)$ having geometric multiplicity of one.

Theorem 2 (see [17]). Consider the following linear fractionalorder system:

$$
{ }_{O}^{C} D_{t}^{q} x(t)=A x(t), \quad \text { with } x(0)=x_{0},
$$


where $x \in \mathfrak{M}^{n}, A \in \mathfrak{M}^{n x n}$, and $q=\left(\begin{array}{llll}q_{1} & q_{2} & \ldots & q_{n}\end{array}\right)^{T}$, with $0<q_{i} \leq 1$ and $q_{i}=n_{i} / d_{i}, \operatorname{gcd}\left(n_{i}, d_{i}\right)=1$.

Let $M$ be the lowest common multiple of the denominators $d_{i}$ 's. The zero solution of system (3) is globally asymptotically stable in the Lyapunov sense if all roots $\lambda$ 's of the equation

$$
\Delta(\lambda)=\operatorname{det}\left(\operatorname{diag}\left(\lambda^{M q_{i}}\right)-A\right)=0
$$

satisfy $|\arg (\lambda)|>\pi / 2 M$.

2.1.2. Stability of the Equilibrium Points. In this section we proceed with commensurate order $q=q_{1}=q_{2}=$ $q_{3}=q_{4}$. Fractional order of the proposed two-stage Colpitts oscillator $(1)$, when $\left(\sigma_{1}, \sigma_{2}, \gamma, \varepsilon\right)=(1.25,1,1.9,1.175)$, has one equilibrium point, $O=(0,0,0,0)$. The Jacobian matrix of system (1), evaluated at the equilibrium point, is

$$
J_{\mid O}=\left(\begin{array}{cccc}
0 & \sigma_{1} \gamma & \sigma_{1} \gamma & \sigma_{1} \\
0 & 0 & 0 & 1 \\
0 & \sigma_{2} \gamma & 0 & \sigma_{2} \\
-1 & -1 & -1 & -\varepsilon
\end{array}\right) .
$$

For $q=(0.96,0.96,0.96,0.96)$ around and to the equilibrium point $O$, the equation $\operatorname{det}\left(\operatorname{diag}\left(\lambda^{M q_{i}}\right)-J_{\mid O}\right)=0$ with $i=1,2,3,4$ and $M=100$ becomes

$$
\begin{aligned}
& \lambda^{384}+\varepsilon \lambda^{288}+\left(1+\sigma_{1}+\sigma_{2}\right) \lambda^{192} \\
& \quad+\left(\sigma_{1} \sigma_{2}+\sigma_{1}+\sigma_{2}\right) \gamma \lambda^{96}+\sigma_{1} \sigma_{2} \gamma^{2}=0 .
\end{aligned}
$$

Thus, for $\gamma=1.1, \min _{i}\{|\arg (\lambda)|\}=0.01596>\pi / 200$ and for $\gamma=1.2, \min _{i}\{|\arg (\lambda)|\}=0.01565<\pi / 200$.

Therefore, on the basis of Theorem 2, system (1) is asymptotically stable at equilibrium point $O$ for $\gamma=1.1$ and unstable for $\gamma=1.2$.

2.2. Hopf Bifurcation. One of the basic differences between the dynamical behavior of fractional-order systems and that of integer-order systems is that the limit set of a trajectory of integer-order system such as a limit cycle is solution for the system under consideration, while in the case of fractionalorder systems, such a limit set of a trajectory may not be solution for this system [18]. In [19], the authors claimed that there are no periodic orbits in fractional-order systems, and in [20], an example is given where the solutions of the system are also not periodic but do converge to periodic signals, confirming in both cases what has been stipulated in [18].

In the present paper, we consider the final state of trajectory that appears at the Hopf bifurcation (after suppression of the transitory state). It is also not a periodic solution of the fractional-order system (1) but attracts nearby solutions.

Let us consider the following four-dimensional fractional-order commensurate system:

$$
D^{q} x=f(\gamma, x),
$$

where $q \in] 0,2\left[, x \in \mathrm{IR}^{4}\right.$, and suppose that $E$ is an equilibrium point of this system. In the integer case $(q=1)$, the stability of $E$ is related to the sign of $\operatorname{Re}\left(\lambda_{i}\right), i=1,2,3,4$, where $\lambda_{i}$ are the eigenvalues of the Jacobian matrix $\partial f /\left.\partial x\right|_{E}$. If $\operatorname{Re}\left(\lambda_{i}\right)<0$ for all $i=1,2,3,4$, then $E$ is locally asymptotically stable. If there exists an $i$ for which $\operatorname{Re}\left(\lambda_{i}\right)>0$, then $E$ is unstable.

To undergo a Hopf bifurcation at the equilibrium point $E$ when $\gamma=\gamma^{*}$, system (7) with $q=1$ must fulfill the following conditions:

(i) The Jacobian matrix must have two pairs of complexconjugate eigenvalues $\lambda_{1,2}(\gamma)=\theta_{1}(\gamma) \pm i \eta_{1}(\gamma)$ and $\lambda_{3,4}(\gamma)=\theta_{2}(\gamma) \pm i \eta_{2}(\gamma)$.

(ii) $\theta_{j}\left(\gamma^{*}\right)=0$, with $j=1,2$;

(iii) $\eta_{j}\left(\gamma^{*}\right) \neq 0$, with $j=1,2$; and finally

(iv) $\partial \theta_{j} /\left.\partial \gamma\right|_{\gamma=\gamma^{*}} \neq 0$.

In the fractional case, the stability of $E$ is related to the sign of $m_{i}(q, \gamma)=q(\pi / 2)-\left|\arg \left(\lambda_{i}(\gamma)\right)\right|$, with $i=1,2,3,4$. If $m_{i}(q, \gamma)<0$ for all $i=1,2,3,4$, the $E$ is locally asymptotically stable. If there exists any $i$ for which $m_{i}(q, \gamma)>0$, then, the equilibrium point $E$ is unstable. So, the function $m_{i}(q, \gamma)$ for fractional-order systems has a similar effect as the real part of eigenvalues in integer system. Therefore, we can extend the Hopf bifurcation condition to the fractional systems by replacing $R_{e}\left(\lambda_{i}\right)$ with $m_{i}(q, \gamma)>0$ as follows, compared with [10]:

(i) $m_{1,2}\left(q, \gamma^{*}\right)=0$,

(ii) $\partial m /\left.\partial \gamma\right|_{\gamma=\gamma^{*}} \neq 0$.

2.3. Hopf Bifurcation versus the Parameter $\beta$ and the Fractional Order $q$. In this subsection, we consider the parameter values $\left(\sigma_{1}, \sigma_{2}, \sigma_{3}\right)=(1.25,1,1.75)$ for the search for the Hopf bifurcation around the equilibrium point $O$.

Figure 1(a) depicts the solution $\left(q^{*}, \gamma^{*}\right)$ of equation $m(q$, $\beta)=0$, while the black curve on Figure $1(\mathrm{~b})$ recalls that $\partial m_{1,2} /\left.\partial \gamma\right|_{\gamma=\gamma^{*}} \neq 0$ for all $0<\gamma^{*}<2$, and for the blue curve in Figure 1(b) it can be noted that $\partial m_{3,4} /\left.\partial \gamma\right|_{\gamma=\gamma^{*}} \neq 0$ for all $0<\gamma^{*}<2$ except for $\gamma^{*}=1.352$. We have $\partial m /\left.\partial q\right|_{q=q^{*}}=\pi / 2 \neq 0$; thus, the proposed fractional-order Hopf bifurcation conditions are verified for all pair $\left(q^{*}, \gamma^{*}\right)$ solutions of $m(q, \beta)=0$, except for $\left(q^{*}, 1.352\right)$.

\section{Numerical Results}

For numerical calculation of fractional-order derivatives, methods defined with (8) to (10) are usually used. The Grünwald-Letnikov (GL) method [14] is given in the following equation:

$$
{ }_{a} D_{t}^{\alpha} f(t)=\lim _{h \rightarrow 0} h^{-\alpha} \sum_{j=0}^{\lfloor(t-\alpha) / h\rfloor}(-1)^{j}\left(\begin{array}{c}
\alpha \\
j
\end{array}\right) f(t-j h),
$$

where $[\cdot]$ indicates the integer part.

The Riemann-Liouville (RL) definition follows as

$$
\begin{array}{r}
{ }_{a} D_{t}^{\alpha} f(t)=\frac{1}{\Gamma(n-\alpha)} \frac{d^{n}}{d t^{n}} \int_{a}^{t} \frac{f(\tau)}{(t-\tau)^{\alpha-n+1}} d \tau, \\
\text { for }(n-1<\alpha<n),
\end{array}
$$




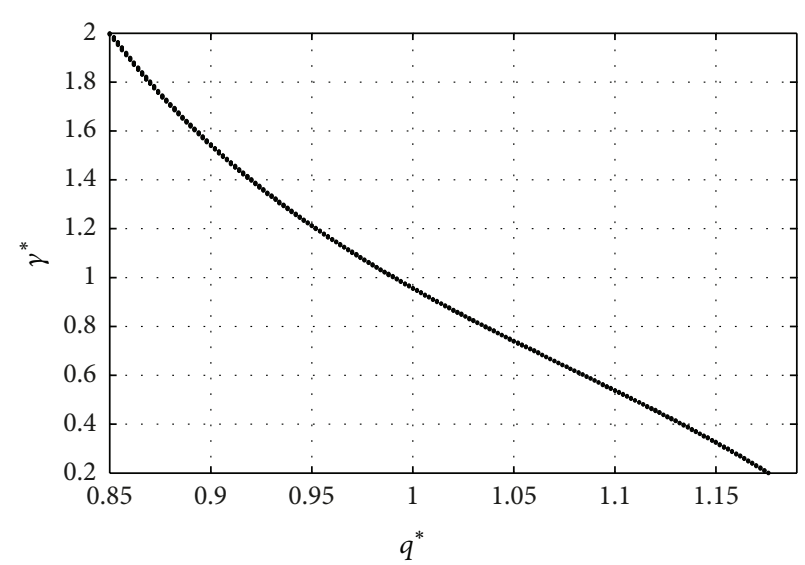

(a)

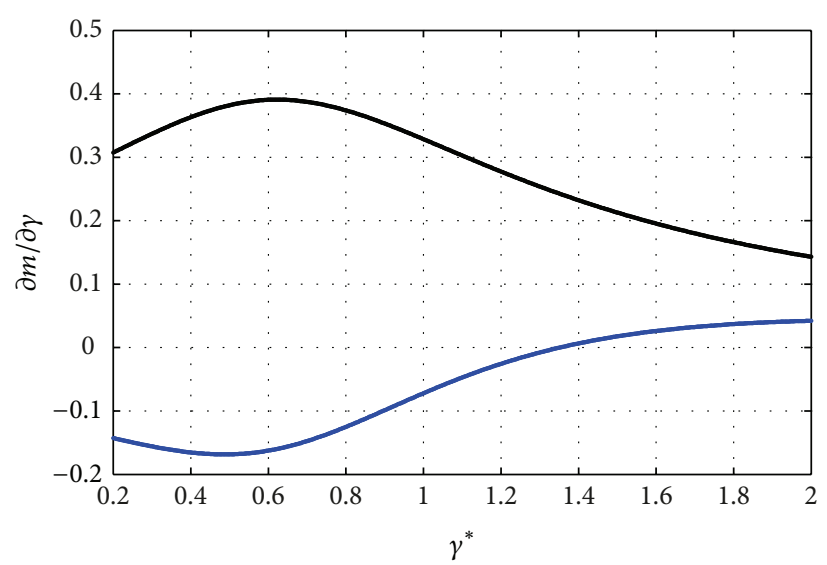

(b)

FIGURE 1: Critical values $\gamma^{*}$ versus the fractional order $q^{*}$. This curve depicts the couples of values for which the Hopf bifurcation occurs in the system.

where $\Gamma(\cdot)$ is the gamma function. The Caputo definition of fractional derivatives can also be recalled as

$$
\begin{array}{r}
{ }_{a} D_{t}^{\alpha} f(t)=\frac{1}{\Gamma(n-\alpha)} \int_{a}^{t} \frac{f^{(n)}(\tau)}{(t-\tau)^{\alpha-n+1}} d \tau, \\
\quad \text { for }(n-1<\alpha<n) .
\end{array}
$$

Based on the fact that for a wide class of functions the three definitions-GL (8), RL (9), and Caputo's (10) are equivalent if $f(a)=0$, we can then use relation (11) derived from the GL definition (8). The new relation for the explicit numerical approximation of $q$ th derivative at the points $k h(k=1,2, \ldots)$ has the following form:

$$
\begin{aligned}
& \left(k-L_{m} / h\right) D_{t_{k}}^{q} f(t) \approx h^{-q} \sum_{j=0}^{h}(-1)^{j}\left(\begin{array}{l}
q \\
j
\end{array}\right) f\left(t_{k}-j\right) \\
& =h^{-q} \sum_{j=0}^{h} C_{j}^{(q)} f\left(t_{k}-j\right),
\end{aligned}
$$

where $L_{m}$ is the "memory length," $t_{k}=k h$, with $h$ the time step of calculation and $C_{j}^{(q)}(j=0,1, \ldots, k)$ the binomial coefficients. For their calculation we can use for instance the following expression:

$$
C_{0}^{(q)}=1, \quad C_{j}^{(q)}=\left(1-\frac{1+q}{j}\right) C_{j-1}^{(q)} .
$$

The binomial coefficients $C_{j}^{(q)}(j=0,1, \ldots, k)$ can also be expressed using a factorial. The gamma function $\Gamma(n)=(n-$ $1)$ ! can allow the generalization of the binomial coefficient to noninteger argument. Thus, relation (12) can be rewritten as follows:

$$
(-1)^{j}\left(\begin{array}{l}
q \\
j
\end{array}\right)=(-1)^{j} \frac{\Gamma(q+1)}{\Gamma(1+1) \Gamma(q-j+1)}=\frac{\Gamma(j-q)}{\Gamma(-q) \Gamma(j+1)} .
$$

3.1. Bifurcation and Chaos versus the Parameter $\gamma$. In this subsection, the dynamical behavior of system (1) is numerically investigated by means of bifurcation diagram and largest Lyapunov exponents, which measure the exponential rates of divergence or convergence of nearby trajectories in phase space. For $\gamma$ taken as control parameter and the following other parameter values, fractional order $q=0.96, \sigma_{1}=1.25$, $\sigma_{2}=1.00$ and $\varepsilon=1.175$, the critical Hopf bifurcation value is localized at $\gamma^{*}=1.150$ (see Figure 2(a)) and confirmed by the largest Lyapunov exponents presented in Figure 2(b). When $\gamma<1.150$, the equilibrium point $O$ is a locally asymptotically stable focus; the neighbors trajectories converge to $O$. This is supported by the negative sign of the largest Lyapunov exponents. For $1.150<\gamma<1.635$, system (1) undergoes a Hopf bifurcation as mentioned above. The fixed point $O$ becomes unstable, and a period-one limit cycle appears. A period-two limit cycle follows for $\gamma \approx 1.635$, leading to a new bifurcation at $\gamma \approx 1.763$, as the system undergoes a period-four bifurcation. This bifurcations scenario continues through a period-height limit cycle for $\gamma \approx 1.791$ up to a critical value of $\gamma \approx 1.820$ corresponding to the appearance of a chaotic attractor. This chaotic behavior is confirmed by the existence of positive largest Lyapunov exponents. Figure 3 depicts the phase portraits presenting routes to chaos according to the abovementioned parameter values.

3.2. Bifurcation and Chaos versus the Fractional Order q. The fractional order $q$ is taken as control parameter, while $\gamma$ is fixed at $\gamma=1.9$. The critical Hopf bifurcation value is localized at $q^{*} \approx 0.8557$, using the previously proposed conditions. The resulting bifurcation diagram (Figure 4(a)) for the second variable of the set of (1) is plotted as a function of the fractional order $q$ and corresponding largest Lyapunov exponents in Figure 4(b).

When $q<0.8557$, the equilibrium point $O$ is a locally asymptotically stable focus confirmed by the negative sign of the largest Lyapunov exponents; the neighbors trajectories converge to this origin. For $q=0.8557$, system (1) undergoes 


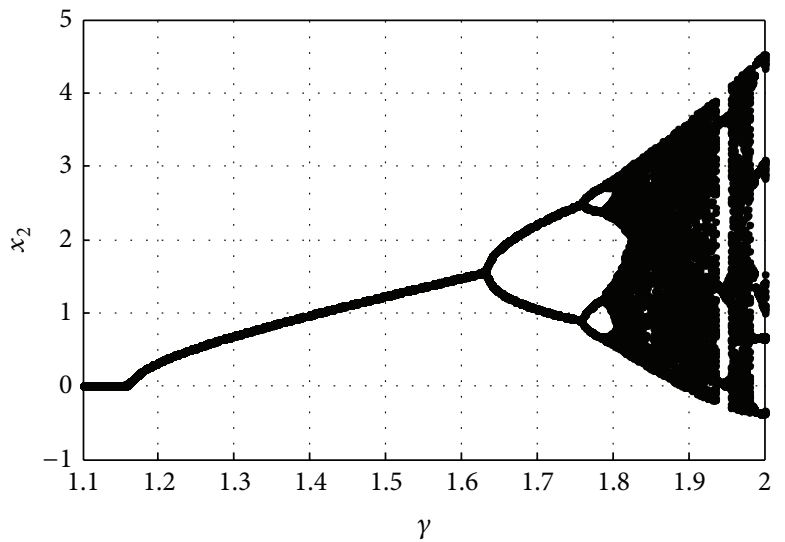

(a)

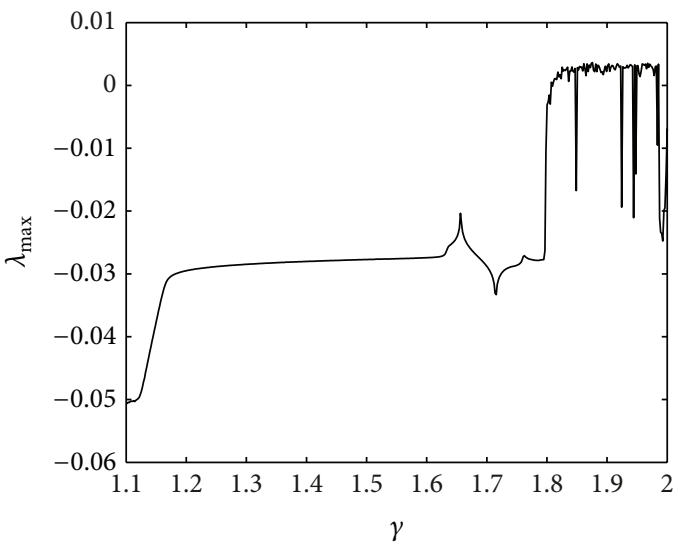

(b)

FIGURE 2: Bifurcation diagram expressing the (a) dynamics of the system variable $x_{2}$ and (b) largest Lyapunov exponent, both as a function of $\gamma$, with $q=0.96$.

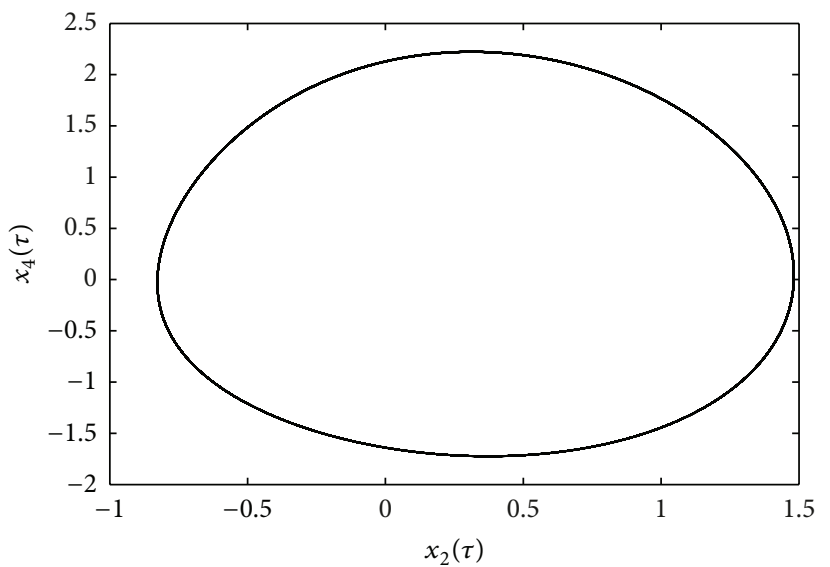

(a)

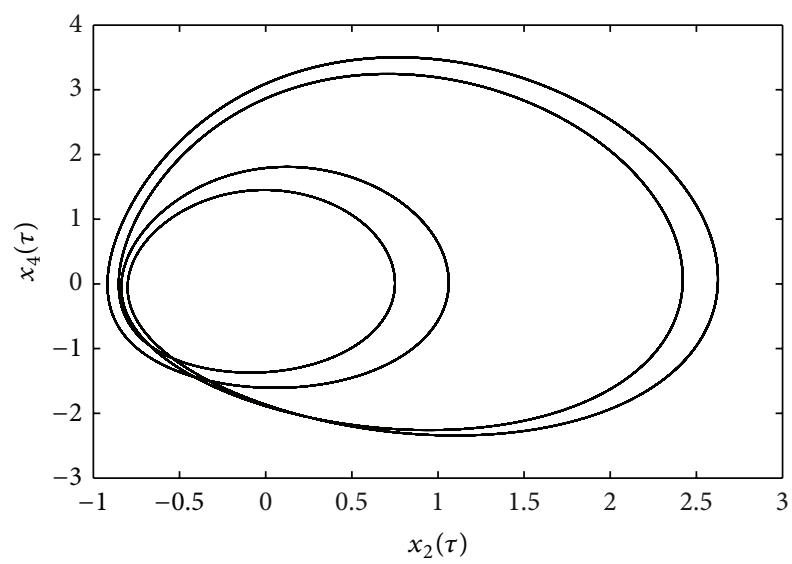

(c)

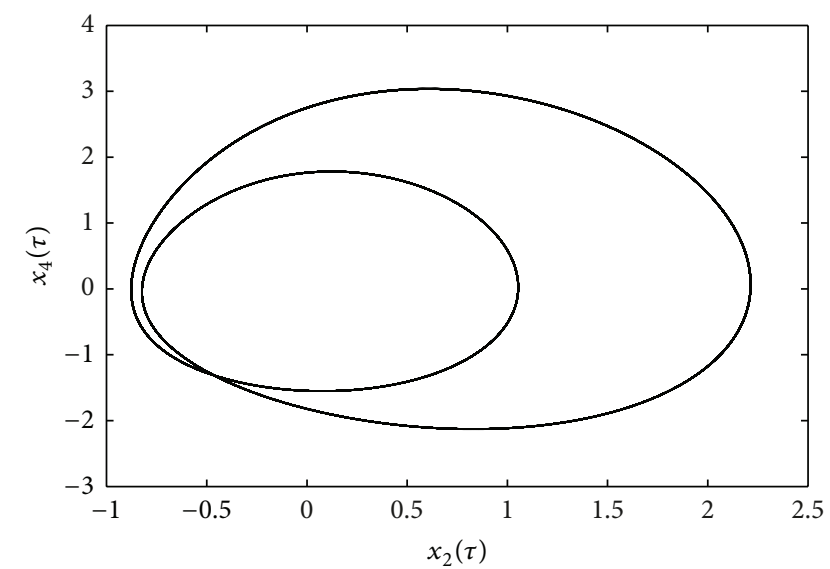

(b)

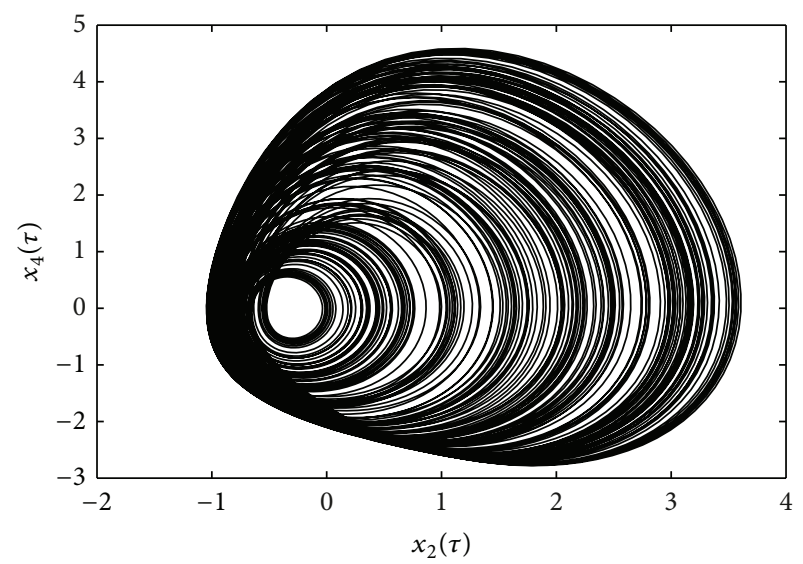

(d)

FIGURE 3: Phase portrait of system (1) for different values of $\gamma$, with $q=0.96$; (a) period-1 for $\gamma=1.3$, (b) period-2 for $\gamma=1.7$, (c) period-4 for $\gamma=1.77$, and (d) chaos for $\gamma=1.9$. 


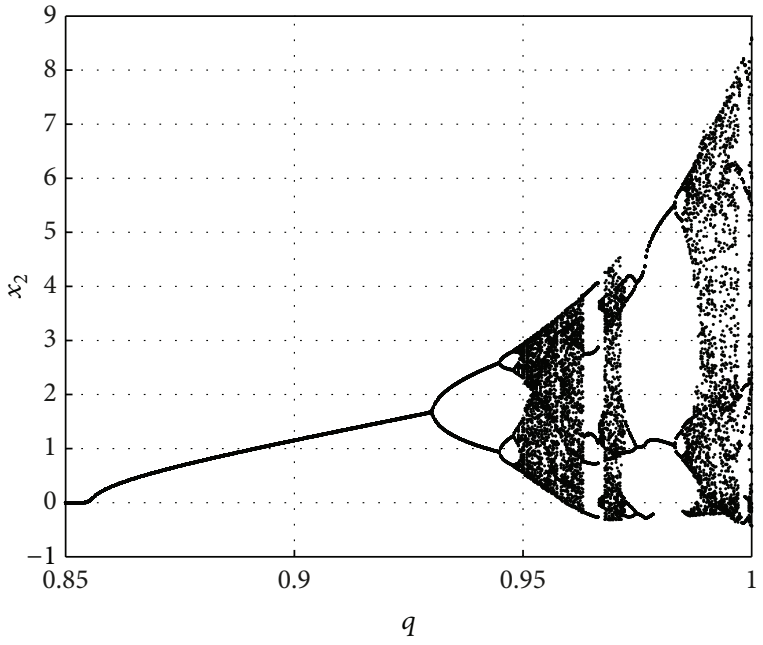

(a)

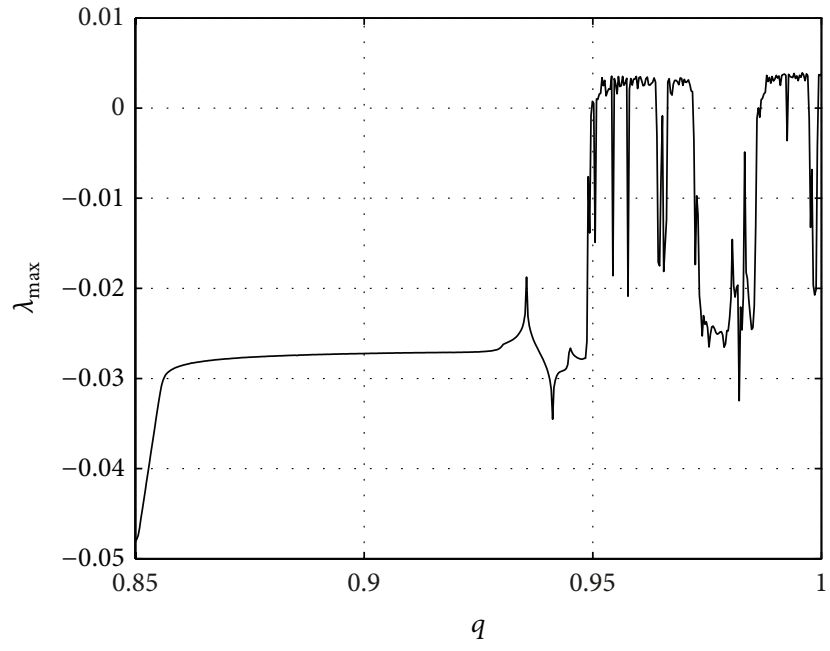

(b)

FIGURE 4: Bifurcation diagram expressing the (a) dynamics of the system variable $x_{2}$ and (b) largest Lyapunov exponent, both as a function of $q$, with $\gamma=1.9$.

a Hopf bifurcation as mentioned above. The fixed point $O$ becomes unstable, and a period-1 limit cycle appears for $0.8557<q<0.9307$. As the fractional order parameter nears the value $q \approx 0.9307$, a new bifurcation occurs for period-2 limit cycle. This is followed by a period-4 limit cycle at $q \approx 0.9454$. This bifurcation scenario continues up to a critical value $q \approx 0.953$ where a chaotic attractor appears, sustained by the existence of positive largest Lyapunov exponents. For a periodic steady state, all spikes in the power spectrum are harmonically related to the fundamental, whereas a broadband noise like power spectrum is associated with a chaotic steady state. The periodicity of the attractor (i.e., total number of frequencies in a wave) is deduced by counting the number of spikes located at the left-hand side of the highest spike (the latter is included). Indeed, we have obtained the complete scenarios to chaos presented in Figure 5. Specifically, the following scenario was observed when monitoring the control parameter: fixed point behavior $\rightarrow$ period- $1 \rightarrow$ period- $2 \rightarrow$ period- $4 \rightarrow$ chaos.

\section{Finite-Time Hybrid Projective Synchronization of Two Fractional-Order Two-Stage Colpitts Oscillators}

4.1. Analytic Results. This section is devoted to the finite-time hybrid projective synchronization of the drive and response commensurate fractional order of a two-stage Colpitts system using a robust fractional nonsingular terminal sliding mode controller, for $q=0.96$. The drive system is defined as follows:

$$
\begin{aligned}
& D^{q} x_{1}=\sigma_{1}\left(x_{4}-\gamma \phi\left(x_{2}+x_{3}\right)\right), \\
& D^{q} x_{2}=x_{4},
\end{aligned}
$$

$$
\begin{aligned}
& D^{q} x_{3}=\sigma_{2}\left(x_{4}-\gamma \phi\left(x_{2}\right)\right), \\
& D^{q} x_{4}=-x_{1}-x_{2}-x_{3}-\varepsilon x_{4} .
\end{aligned}
$$

The drive system (14) can be written as well as

$$
D^{q} x_{i}=f_{i}\left(t, x_{i}\right), \quad i=1, \ldots, 4 .
$$

Accordingly, the response system takes the following form:

$$
D^{q} y_{i}=f_{i}\left(t, y_{i}\right)+\Delta f_{i}\left(t, y_{i}\right)+d_{i}+u_{i}, \quad i=1, \ldots, 4,
$$

where $u_{i} \in \mathrm{IR}^{4}$ represents the nonlinear controllers and $\Delta f_{i}\left(t, y_{i}\right)$ and $d_{i} \in \mathrm{IR}^{4}$ represent unknown model uncertainty and external disturbances of the system. By subtracting (15) from (16) and setting

$$
e_{i}=a_{i} y_{i}-x_{i}, \quad i=1, \ldots, 4,
$$

the following set of equations defining the errors is obtained:

$$
D^{\alpha} e_{i}=a_{i}\left(f_{i}\left(t, y_{i}\right)+\Delta f_{i}\left(t, y_{i}\right)+d_{i}+u_{i}\right)-f_{i}\left(t, x_{i}\right) .
$$

Theorem 3 (see [27]). Let $x=0$ be an equilibrium point for the nonautonomous fractional-order system $D^{\alpha} x=w(t, x)$, where $w(t, x)$ satisfies the Lipschitz condition with Lipschitz constant $l>0$ and $\alpha \in\left(\begin{array}{ll}0 & 1\end{array}\right)$. Assume that there exists a Lyapunov function $V(t, x)$ satisfying

$$
\begin{gathered}
\alpha_{1}\|x\|^{d} \leq V(t, x) \leq \alpha_{2}\|x\|, \\
\dot{V}(t, x) \leq \alpha_{3}\|x\|,
\end{gathered}
$$

where $\alpha_{1}, \alpha_{2}, \alpha_{3}$, and $d$ are positive constants. Then the equilibrium point of the system $D^{\alpha} x=w(t, x)$ is Mittag-Leffler stable. 


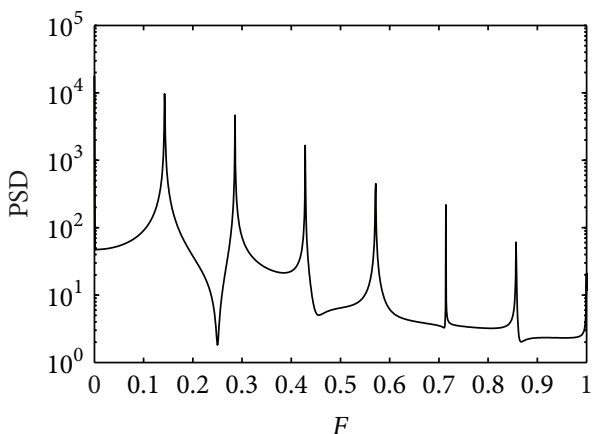

(a)

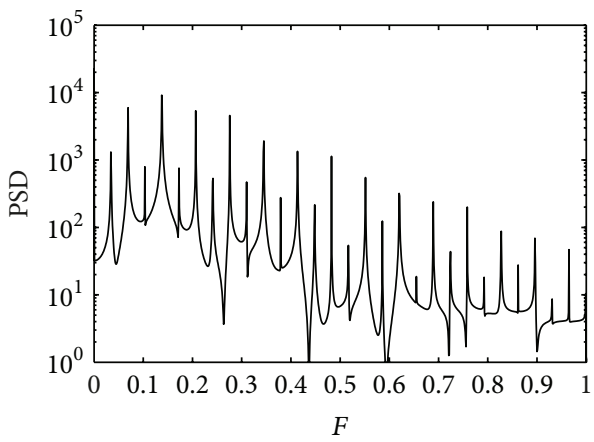

(c)

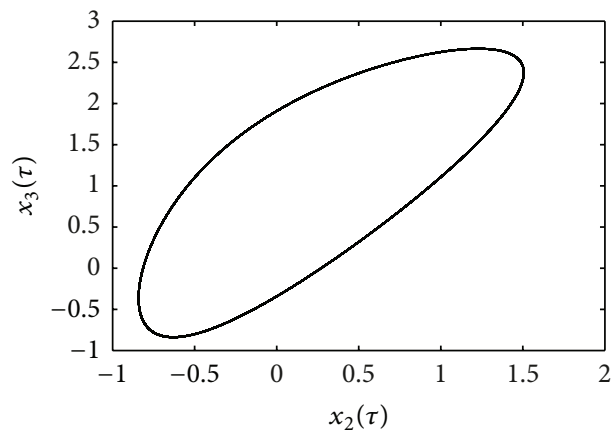

(e)

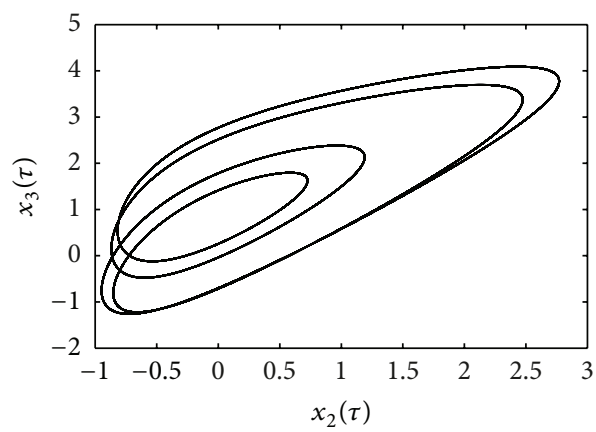

(g)

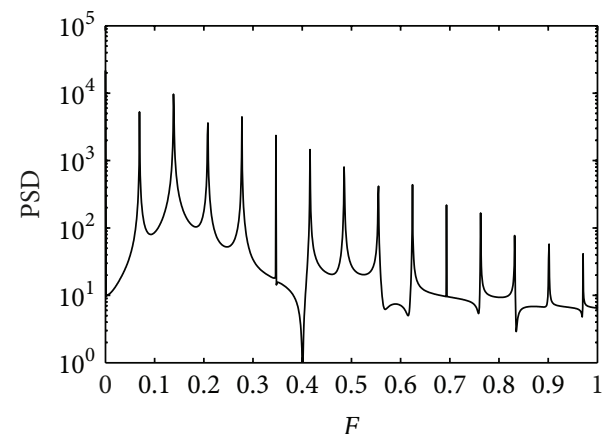

(b)

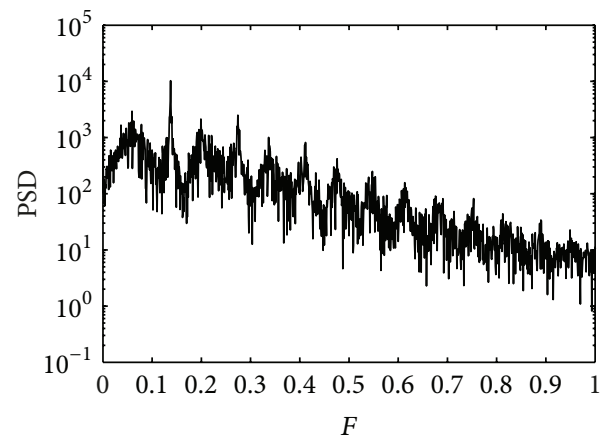

(d)

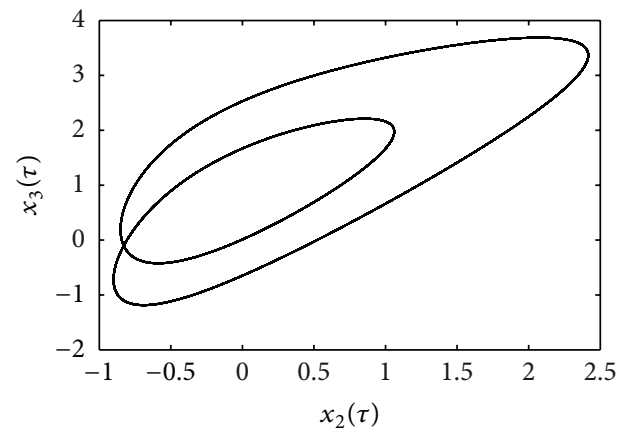

(f)

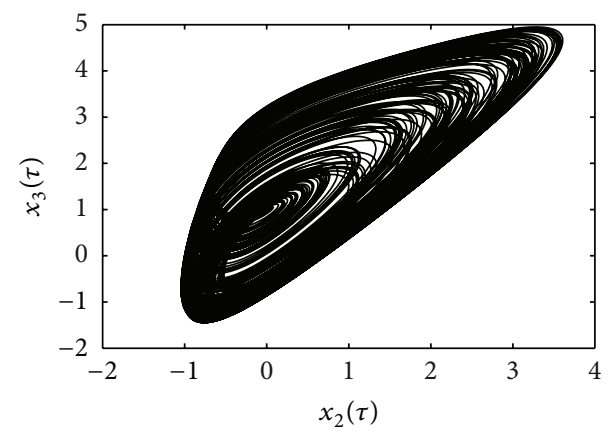

(h)

FIGURE 5: Phase portrait of system (1) for different values of $q$, with $\gamma=1.9$ and corresponding power spectra: ((a) and (e)) period-1 for $q=0.92$, ((b) and (f)) period-2 for $q=0.94,((\mathrm{c})$ and $(\mathrm{g}))$ period -4 for $q=0.947$, and ((d) and (h)) chaos for $q=0.96$. 
Assumption 4. The uncertainty terms $\Delta f_{i}\left(t, y_{i}\right)$ and external disturbance $d_{i}$ are bounded by $\left|\Delta f_{i}\left(t, y_{i}\right)\right| \leq \delta_{i}$ and $\left|d_{i}\right| \leq \beta_{i}$, $i=1, \ldots, 4$, where $\delta_{i}$ and $\beta_{i}$ are positive constants.

Lemma 5. Assume $c, b$, and $0<n<1$ are real numbers; then the following inequality holds: $(|c|+|b|)^{n} \leq|c|^{n}+|b|^{n}$.

4.1.1. Main Results. In this subsection a modified nonsingular terminal sliding surface is proposed as

$$
\begin{array}{r}
S_{i}(t)=D^{q-1} e_{i}+D^{q-2}\left(K_{i} e_{i}+K_{i}\left|a_{i} e_{i}\right|^{\mu} \operatorname{sign}\left(e_{i}\right)\right), \\
i=1, \ldots, 4,
\end{array}
$$

where $K_{i}$ are the sliding surface parameters to be introduced later and $a_{i}$ are the scaling factor to content in synchronization error. The nonsingular terminal sliding surface as defined in (20) present a major advantage on that proposed in [27], namely its dependence on scaling factor $a_{i}$ no matter the initial conditions which, allows to improve the synchronization time between the driven system and response system. For the existence of the sliding mode it is necessary and sufficient that $S_{i}(t)=0$ and $\dot{S}_{i}(t)=0$ [27]. Therefore, the dynamics of the proposed nonsingular terminal sliding mode can be obtained as

$$
D^{q} e_{i}=-D^{q-1}\left(K_{i} e_{i}+K_{i}\left|a_{i} e_{i}\right|^{\mu} \operatorname{sign}\left(e_{i}\right)\right), \quad i=1, \ldots, 4 .
$$

Theorem 6. The system (20) is finite time stable and its trajectories converge to the equilibrium $e(t)=0$ in a finitetime, $t_{s 1}$, determined by

$$
t_{s 1} \leq \frac{1}{K(1-\mu)}\left[\ln \left(1+\frac{\|e(0)\|^{1-\mu}}{|a|^{\mu}}\right)\right],
$$

where $K=\min \left(K_{i}\right)$ and $a=\min \left(a_{i}\right)$.

Proof. Consider the candidate following positive definite Lyapunov function:

$$
V_{1}=\sum_{i=1}^{4}\left|e_{i}\right|=\|e\|
$$

The time derivative of the Lyapunov function along the trajectories of (21) is

$$
\begin{aligned}
\dot{V}_{1} & =\sum_{i=1}^{4} \operatorname{sign}\left(e_{i}\right) \dot{e}_{i} \\
& =\sum_{i=1}^{4} \operatorname{sign}\left(e_{i}\right)\left(D^{1-q}\left(D^{q} e_{i}\right)\right) \\
& =\sum_{i=1}^{4} \operatorname{sign}\left(e_{i}\right)\left[D^{1-q}\left(-D^{q-1}\left(K_{i} e_{i}+K_{i}\left|a_{i} e_{i}\right|^{\mu} \operatorname{sign}\left(e_{i}\right)\right)\right)\right]
\end{aligned}
$$

$$
\begin{aligned}
& =-\sum_{i=1}^{4} \operatorname{sign}\left(e_{i}\right)\left(K_{i} e_{i}+K_{i}\left|a_{i} e_{i}\right|^{\mu} \operatorname{sign}\left(e_{i}\right)\right) \\
& \leq-K\left(\sum_{i=1}^{4}\left|e_{i}\right|+|a|^{\mu} \sum_{i=1}^{4}\left|e_{i}\right|^{\mu}\right) .
\end{aligned}
$$

Using Lemma 5, one can obtain

$$
\dot{V}_{1} \leq-K\left(\|e\|+|a|^{\mu}\|e\|^{\mu}\right) \leq-K\|e\| .
$$

Hence, according to Theorem 3, the error system (21) will converge to zero asymptotically. In order to show that the sliding motion occurs in finite time, we can obtain the convergence time as follows.

From inequality (25) we have

$$
\frac{d\|e\|}{d t} \leq-K\left(\|e\|+|a|^{\mu}\|e\|^{\mu}\right) .
$$

What leads us to

$$
d t \leq-\frac{1}{K(1-\mu)} \frac{d\|e\|^{1-\mu}}{K\left(\|e\|^{1-\mu}+|a|^{\mu}\right)} .
$$

Taking integral of both sides of (27) from 0 to $t_{s 1}$ and letting $e\left(t_{S_{1}}\right)=0$, we have

$$
t_{s 1} \leq \frac{1}{K(1-\mu)}\left[\ln \left(1+\frac{\|e(0)\|^{1-\mu}}{|a|^{\mu}}\right)\right] .
$$

Therefore, the state trajectories of the error system (21) will converge to $e(t)=0$ in the finite time $t_{s 1} \leq(1 / K(1-\mu))[\ln (1+$ $\left.\left.\left(\|e(0)\|^{1-\mu} /|a|^{\mu}\right)\right)\right]$. This completes the proof.

A control law which forces the error trajectories to go onto the sliding surface within a finite time and remain on it forever is designed as follows:

$$
\begin{array}{r}
u_{i}=\frac{1}{a_{i}}\left(f_{i}\left(t, x_{i}\right)-\varepsilon_{i} S_{i}-\left(\left|a_{i}\right|\left(\delta_{i}+\beta_{i}\right)+\varepsilon_{i}\left|S_{i}\right|^{\eta}\right) \operatorname{sign}\left(S_{i}\right)\right. \\
\left.-D^{q-1}\left(K_{i} e_{i}+K_{i}\left|a_{i} e_{i}\right|^{\mu} \operatorname{sign}\left(e_{i}\right)\right)\right)-f_{i}\left(t, y_{i}\right)
\end{array}
$$

$i=1, \ldots, 4$, where $K_{i}$ are the sliding surface parameters to be introduced later, $a_{i}$ are the scaling factor to content in synchronization error, and $\delta_{i}$ and $\beta_{i}$ are positive constants.

Theorem 7. If the error system (18) is controlled with control law (29), then the states of the system will move toward the sliding surface and will approach the sliding surface $S_{i}(t)=0$ in a finite time, $t_{s 2}$, given by

$$
t_{s 2} \leq \frac{1}{\varepsilon(1-\eta)} \ln \left(\|S(0)\|^{1-\eta}+1\right) .
$$

Proof. Choosing a Lyapunov function in the form of

$$
V_{2}=\|S(t)\|=\sum_{i=1}^{4}\left|S_{i}\right|
$$


and taking the time derivative, one has

$$
\dot{V}_{2}=\sum_{i=1}^{4} \operatorname{sign}\left(S_{i}\right) \dot{S}_{i}
$$

Inserting (20) into (32), we have

$$
\dot{V}_{2}=\sum_{i=1}^{4} \operatorname{sign}\left(S_{i}\right)\left[D^{q} e_{i}+D^{q-1}\left(K_{i} e_{i}+K_{i}\left|a_{i} e_{i}\right|^{\mu} \operatorname{sign}\left(e_{i}\right)\right)\right] \text {. }
$$

Inserting (18) into (33), we have

$$
\begin{gathered}
\dot{V}_{2}=\sum_{i=1}^{4} \operatorname{sign}\left(S_{i}\right) \\
\times\left[a_{i}\left(f_{i}\left(t, y_{i}\right)+\Delta f_{i}\left(t, y_{i}\right)+d_{i}+u_{i}\right)-f_{i}\left(t, x_{i}\right)\right. \\
\left.+D^{q-1}\left(K_{i} e_{i}+K_{i}\left|a_{i} e_{i}\right|^{\mu} \operatorname{sign}\left(e_{i}\right)\right)\right] .
\end{gathered}
$$

Using expression $\operatorname{sign}^{2}\left(S_{i}\right)=1$ into (34), we obtain

$$
\begin{gathered}
\dot{V}_{2} \leq \sum_{i=1}^{4} \operatorname{sign}\left(S_{i}\right)\left[a_{i} f_{i}\left(t, y_{i}\right)+a_{i} u_{i}-f_{i}\left(t, x_{i}\right)\right. \\
\left.+D^{q-1}\left(K_{i} e_{i}+K_{i}\left|a_{i} e_{i}\right|^{\mu} \operatorname{sign}\left(e_{i}\right)\right)\right] \\
+\sum_{i=1}^{4}\left(\left|a_{i}\right|\left|\Delta f_{i}\left(t, y_{i}\right)\right|+\left|a_{i}\right|\left|d_{i}\right|\right) \operatorname{sign}^{2}\left(S_{i}\right)
\end{gathered}
$$

On the basis of Assumption 4, we get

$$
\begin{gathered}
\dot{V}_{2} \leq \sum_{i=1}^{4} \operatorname{sign}\left(S_{i}\right)\left[a_{i} f_{i}\left(t, y_{i}\right)+a_{i} u_{i}-f_{i}\left(t, x_{i}\right)\right. \\
\left.+D^{q-1}\left(K_{i} e_{i}+K_{i}\left|a_{i} e_{i}\right|^{\mu} \operatorname{sign}\left(e_{i}\right)\right)\right] \\
+\sum_{i=1}^{4}\left(\left|a_{i}\right|\left|\delta_{i}\right|+\left|a_{i}\right|\left|\beta_{i}\right|\right) \operatorname{sign}^{2}\left(S_{i}\right),
\end{gathered}
$$

what leads to

$$
\begin{aligned}
\dot{V}_{2} \leq \sum_{i=1}^{4} \operatorname{sign}\left(S_{i}\right)[ & a_{i} f_{i}\left(t, y_{i}\right)+a_{i} u_{i}-f_{i}\left(t, x_{i}\right) \\
& +\left(\left|a_{i}\right|\left|\delta_{i}\right|+\left|a_{i}\right|\left|\beta_{i}\right|\right) \operatorname{sign}\left(S_{i}\right) \\
& \left.+D^{q-1}\left(K_{i} e_{i}+K_{i}\left|a_{i} e_{i}\right|^{\mu} \operatorname{sign}\left(e_{i}\right)\right)\right] .
\end{aligned}
$$

Introducing (29) into (37), we have

$$
\begin{gathered}
\dot{V}_{2} \leq \sum_{i=1}^{4} \operatorname{sign}\left(S_{i}\right)\left[-\varepsilon_{i} S_{i}-\varepsilon_{i}\left|S_{i}\right|^{\eta} \operatorname{sign}\left(S_{i}\right)\right] \\
\dot{V}_{2} \leq \sum_{i=1}^{4}\left[-\varepsilon_{i} S_{i}-\varepsilon_{i}\left|S_{i}\right|^{\eta}\right] .
\end{gathered}
$$

Using Lemma 5, one can obtain

$$
\dot{V}_{2} \leq-\varepsilon\left(\|S(t)\|+\|S(t)\|^{\eta}\right)
$$

where $\varepsilon=\min \left(\varepsilon_{i}\right)$.

Hence, according to Theorem 3, the error system (18) will converge to $S_{i}(t)=0$ asymptotically.

In order to show that the sliding motion occurs in finite time, we can obtain the convergence time as follows.

From inequality (39), we have

$$
d t \leq-\frac{d\|S(t)\|}{\varepsilon\left(\|S(t)\|+\|S(t)\|^{\eta}\right)}
$$

what leads us to

$$
d t \leq-\frac{1}{\varepsilon(1-\eta)} \frac{d\|S(t)\|^{1-\eta}}{\left(\|S(t)\|^{1-\eta}+1\right)} .
$$

Taking integral of both sides of (41) from 0 to $t_{s 2}$ and letting $e\left(t_{s 2}\right)=0$, we have

$$
t_{s 2} \leq \frac{1}{\varepsilon(1-\eta)} \ln \left(\|S(0)\|^{1-\eta}+1\right)
$$

Remark 8. According to Theorems 6 and 7, the sliding mode control law (29) and the sliding surface (20) can make the response system (16) reach the drive system (15) in the finitetime $T_{s}=t_{s 1}+t_{s 2}$.

4.2. Numerical Results. The drive and response system (14) and (16) are numerically integrated with the parameter values $\sigma_{1}=1.25, \sigma_{2}=1.00, \gamma=1.9, \varepsilon=1.175$, and $q=0.96$; the uncertainties and external disturbance in (16) are selected by

$$
\begin{aligned}
& \Delta f_{1}\left(t, y_{1}\right)+d_{1}=0.1 \sin \left(0.1 \pi y_{1}\right)+0.1 \cos (0.1 t), \\
& \Delta f_{2}\left(t, y_{2}\right)+d_{2}=0.1 \cos \left(0.1 \pi y_{2}\right)+0.1 \sin (0.1 t), \\
& \Delta f_{3}\left(t, y_{3}\right)+d_{3}=0.1 \sin \left(0.1 \pi y_{3}\right)+0.1 \cos (0.1 t), \\
& \Delta f_{4}\left(t, y_{4}\right)+d_{4}=0.1 \cos \left(0.1 \pi y_{4}\right)+0.1 \sin (0.1 t) .
\end{aligned}
$$

Control laws (29) use the nonsingular surface defined at (20) and the rest of functions of (29) are chosen as:

$$
\begin{aligned}
& f_{i}(t, x)=\left(\begin{array}{l}
f_{1}\left(t, x_{1}\right) \\
f_{2}\left(t, x_{2}\right) \\
f_{3}\left(t, x_{3}\right) \\
f_{4}\left(t, x_{4}\right)
\end{array}\right)=\left(\begin{array}{c}
\sigma_{1}\left(x_{4}-\gamma \phi\left(x_{2}+x_{3}\right)\right) \\
x_{4} \\
\sigma_{2}\left(x_{4}-\gamma \phi\left(x_{2}\right)\right) \\
-x_{1}-x_{2}-x_{3}-\varepsilon x_{4}
\end{array}\right), \\
& f_{i}(t, y)=\left(\begin{array}{l}
f_{1}\left(t, y_{1}\right) \\
f_{2}\left(t, y_{2}\right) \\
f_{3}\left(t, y_{3}\right) \\
f_{4}\left(t, y_{4}\right)
\end{array}\right)=\left(\begin{array}{c}
\sigma_{1}\left(y_{4}-\gamma \phi\left(y_{2}+y_{3}\right)\right) \\
y_{4} \\
\sigma_{2}\left(y_{4}-\gamma \phi\left(y_{2}\right)\right) \\
-y_{1}-y_{2}-y_{3}-\varepsilon y_{4}
\end{array}\right),
\end{aligned}
$$



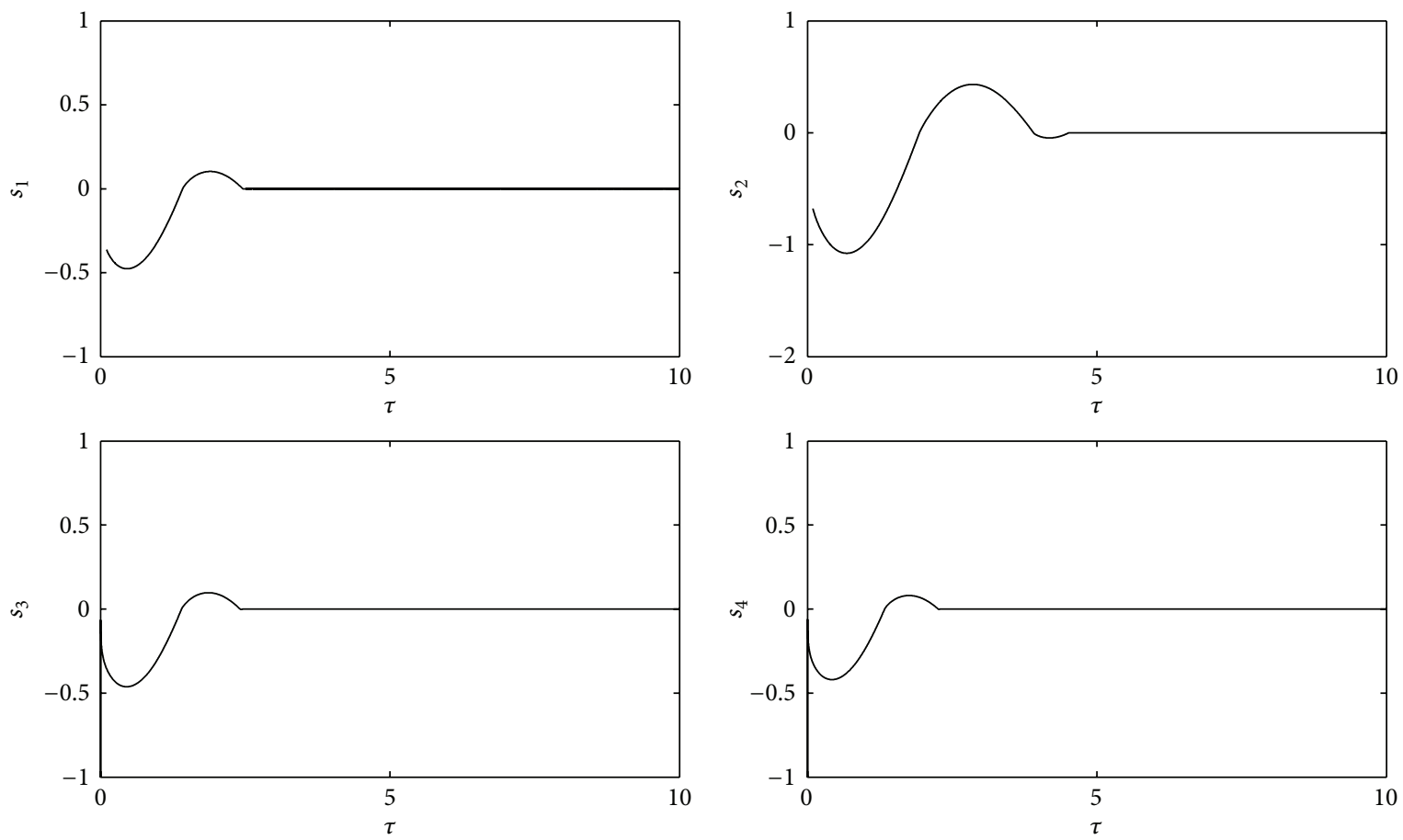

(a)
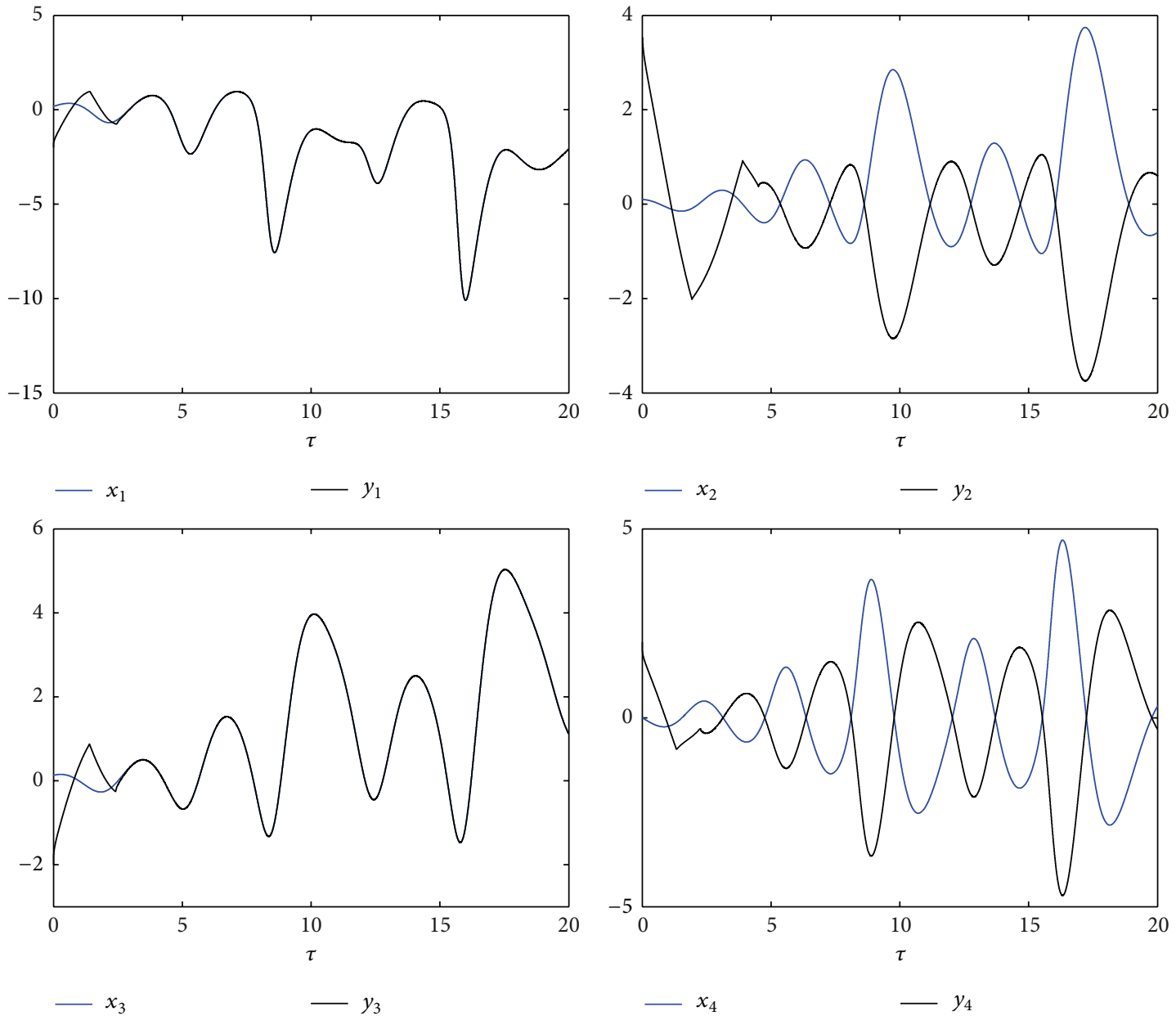

(b)

FIGURE 6: Continued. 

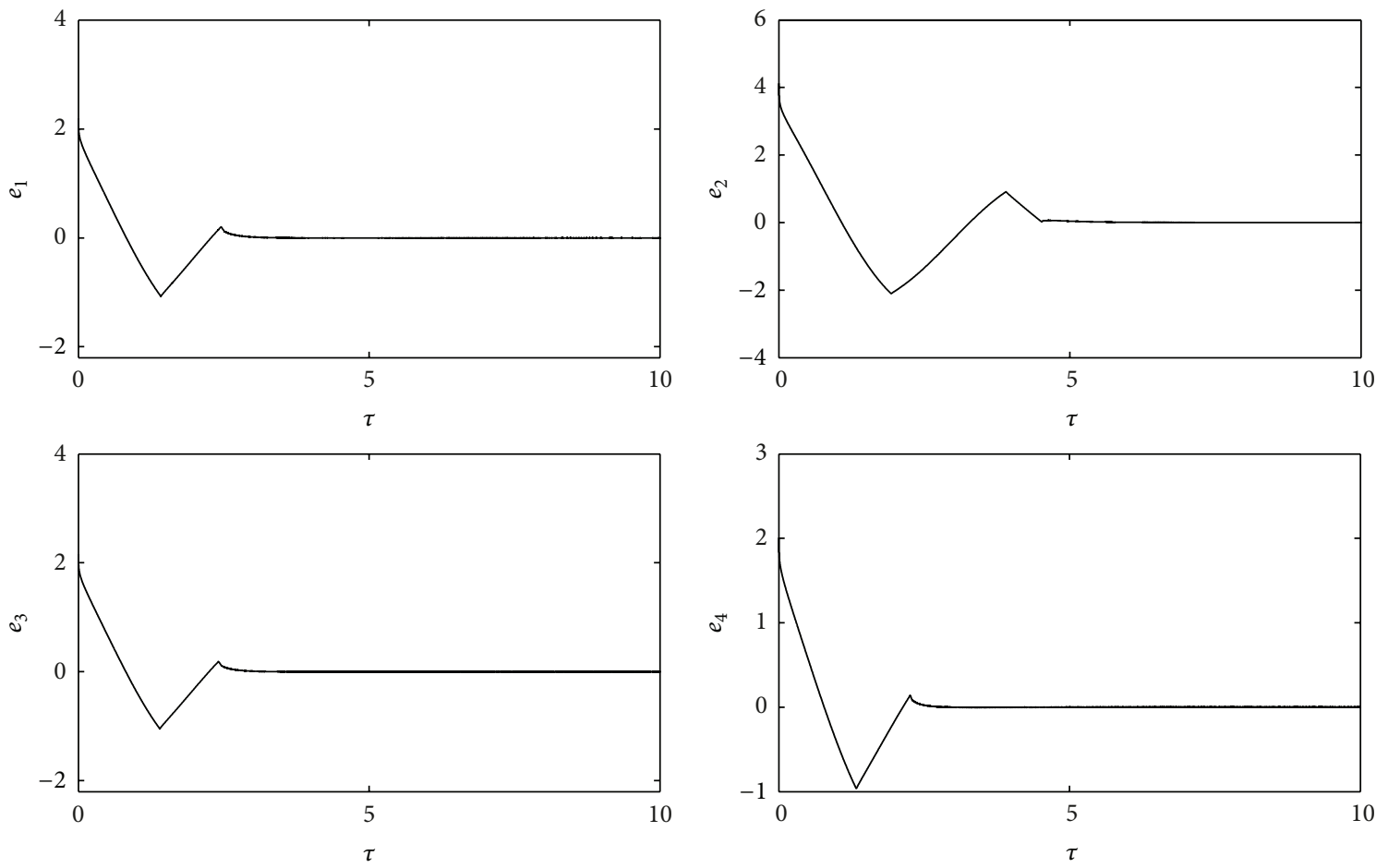

(c)

FIGURE 6: (a) Time evolution of the sliding mode surface, showing the (b) synchronization process between the two coupled chaotic oscillators starting from different initial condition and (c) synchronization errors obtained for $\sigma_{1}=1.25, \sigma_{2}=1.00, \gamma=1.9, \varepsilon=1.175, q=0.96$, $k_{1}=k_{2}=k_{3}=k_{4}=0.15, \varepsilon_{1}=\varepsilon_{2}=\varepsilon_{3}=\varepsilon_{4}=0.5, \eta=\mu=0.95, a_{1}=a_{3}=2$, and $a_{2}=a_{4}=-2$.

$$
\begin{aligned}
S_{i}(t) & =\left(\begin{array}{l}
S_{1}(t) \\
S_{2}(t) \\
S_{3}(t) \\
S_{4}(t)
\end{array}\right) \\
& =\left(\begin{array}{l}
D^{q-1} e_{1}+D^{q-2}\left(K_{1} e_{1}+K_{1}\left|a_{1} e_{1}\right|^{\mu} \operatorname{sign}\left(e_{1}\right)\right) \\
D^{q-1} e_{2}+D^{q-2}\left(K_{2} e_{2}+K_{2}\left|a_{2} e_{2}\right|^{\mu} \operatorname{sign}\left(e_{2}\right)\right) \\
D^{q-1} e_{3}+D^{q-2}\left(K_{3} e_{3}+K_{3}\left|a_{3} e_{3}\right|^{\mu} \operatorname{sign}\left(e_{3}\right)\right) \\
D^{q-1} e_{4}+D^{q-2}\left(K_{4} e_{4}+K_{4}\left|a_{4} e_{4}\right|^{\mu} \operatorname{sign}\left(e_{4}\right)\right)
\end{array}\right),
\end{aligned}
$$

and the parameters of (29) are selected as follows: $k_{1}=k_{2}=$ $k_{3}=k_{4}=0.15, \varepsilon_{1}=\varepsilon_{2}=\varepsilon_{3}=\varepsilon_{4}=0.5, \eta=\mu=0.95$, $a_{1}=a_{3}=2$, and $a_{2}=a_{4}=-2$. The initial conditions are given as $\left[\begin{array}{llll}0.2 & 0.12 & 0.15 & 0.01\end{array}\right]$ for the driver and $\left[\begin{array}{lll}-1 & 2 & -\end{array}\right.$ 1 1] for the response. The analytics time of synchronization is $T_{a}=61.17+29.85$, whereas in Figure 6 we show that the numerical time of synchronization is $T_{n}=3.13+6.13$. These results confirm our theoretical result. Figure 7 displays MSQR $=\sqrt{\sum_{i=1}^{4} e_{i}^{2}}$ for the parameters selects as follows: $k_{1}=k_{2}=k_{3}=k_{4}=0.15, \varepsilon_{1}=\varepsilon_{2}=\varepsilon_{3}=\varepsilon_{4}=0.5$, $\eta=\mu=0.95$, and $a_{i}$ are given in the key. The initial conditions are as follows: $\left[\begin{array}{llll}0.2 & 0.12 & 0.75 & 0.1\end{array}\right]$ for the master and $\left[\begin{array}{llll}0 & 0 & 0 & 0\end{array}\right]$ for the slave. Figure 7 shows us that when $a_{i}$ is far from one, the synchronization time turns fast to zero; this decreasing numerical time of synchronization justifies

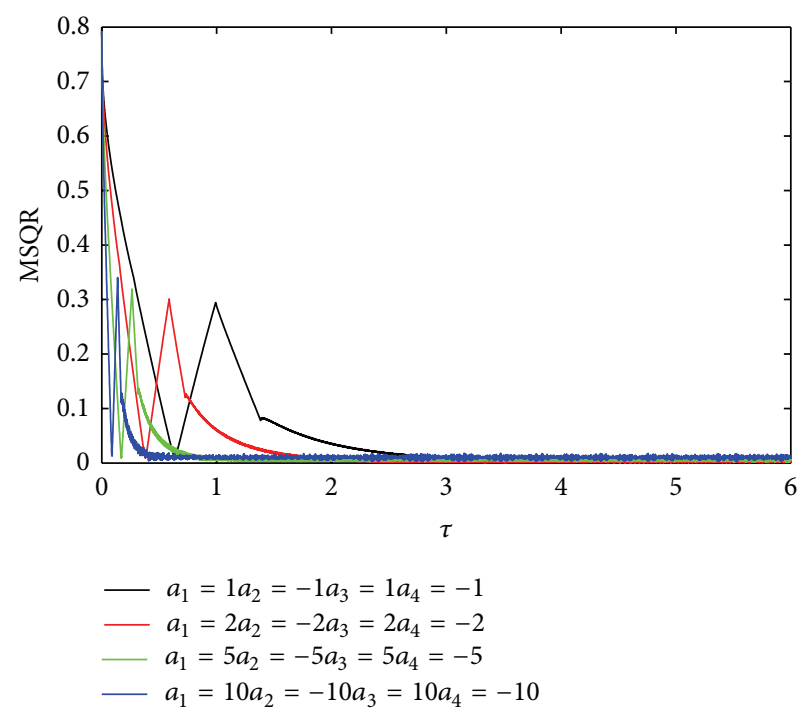

FIGURE 7: Time evolution of MSQR $=\sqrt{\sum_{i=1}^{4} e_{i}^{2}}$.

the decreasing analytical time of synchronization which is, respectively, $t_{a}=120.55, t_{a}=83.58, t_{a}=53.95$, and $t_{a}=41.89$ for $\left|a_{i}\right|=1,\left|a_{i}\right|=2,\left|a_{i}\right|=5$, and $\left|a_{i}\right|=10$. 


\section{Conclusion}

In this paper the dynamics and synchronization of a proposed four-dimensional fractional-order two-stage Colpitts oscillator have been investigated using analytical and numerical methods. The analytic method proved the existence of the Hopf bifurcation as well as the beach of the control parameter for which the system is stable. On the basis of fractional Lyapunov stability theory we determined with success the conditions under which the synchronization of two systems is achieved. For numerical simulation we used the GrünwaldLetnikov method, the largest Lyapunov exponents, and the bifurcation diagrams to show the period-doubling bifurcation routes to chaos as well as the Hopf bifurcation. The numerical analysis validates the conditions of Hopf bifurcation. For the finite-time hybrid projective synchronization the numerical investigation validates also the analytic conditions which achieve synchronization. Numerical simulations have been used to show the effectiveness of the proposed synchronization techniques.

\section{Conflict of Interests}

The authors declare that there is no conflict of interests regarding the publication of this paper.

\section{References}

[1] R. Hilfer, Applications of Fractional Calculus in Physics, World Scientific, New Jersey, NJ, USA, 2001.

[2] R. C. Koeller, "Application of fractional calculus to the theory of viscoelasticity," Journal of Applied Mechanics, vol. 51, no. 2, pp. 299-307, 1984.

[3] H. H. Sun, A. A. Abdelwahab, and B. Onaral, "Linear approximation of transfer function with a pole of fractional order," IEEE Transactions on Automatic Control, vol. 29, no. 5, pp. 441-444, 1984.

[4] O. Heaviside, Electromagnetic Theory, Chelsea, New York, NY, USA, 1971.

[5] I. Grigorenko and E. Grigorenko, "Chaotic dynamics of the fractional Lorenz system," Physical Review Letters, vol. 91, no. 3, Article ID 034101, 2003.

[6] K. Sun and J. C. Sprott, "Bifurcations of fractional-order diffusionless lorenz system," Electronic Journal of Theoretical Physics, vol. 6, no. 22, pp. 123-134, 2009.

[7] P. Zhou and W. Zhu, "Function projective synchronization for fractional-order chaotic systems," Nonlinear Analysis, vol. 12, no. 2, pp. 811-816, 2011.

[8] D. Chen, Y. Q. Chen, and H. Sheng, "Fractional variational optical flow model for motion estimation," in Proceedings of the 4th IFAC Workshop Fractional Differentiation and Its Application, pp. 18-20, Badajoz, Spain, October 2010.

[9] J. C. Gutiérrez-Vega and C. López-Mariscal, "Nondiffracting vortex beams with continuous orbital angular momentum order dependence," Journal of Optics A, vol. 10, no. 1, Article ID 015009, pp. 1-8, 2008.

[10] M.-S. Abdelouahab, N.-E. Hamri, and J. Wang, "Hopf bifurcation and chaos in fractional-order modified hybrid optical system," Nonlinear Dynamics, vol. 69, no. 1-2, pp. 275-284, 2012.
[11] P. Zhou, "Control and synchronization of the fractional-order Lorenz chaotic system via fractional-order derivative," Mathematical Problems in Engineering, vol. 2012, Article ID 214169, 14 pages, 2012.

[12] P. Zhou, R. Ding, and Y. X. Cao, "Multi drive-one response synchronzation for fractional-order chaotic systems," Nonlinear Dynamics, vol. 70, no. 2, pp. 1263-1271, 2012.

[13] S. Wang, Y. Yu, and M. Diao, "Hybrid projective synchronization of chaotic fractional order systems with different dimensions," Physica A, vol. 389, no. 21, pp. 4981-4988, 2010.

[14] S. H. Hosseinnia, R. Ghaderi, A. Ranjbar N., M. Mahmoudian, and S. Momani, "Sliding mode synchronization of an uncertain fractional order chaotic system," Computers and Mathematics with Applications, vol. 59, no. 5, pp. 1637-1643, 2010.

[15] E. Ahmed, A. M. A. El-Sayed, and H. A. A. El-Saka, "Equilibrium points, stability and numerical solutions of fractionalorder predator-prey and rabies models," Journal of Mathematical Analysis and Applications, vol. 325, no. 1, pp. 542-553, 2007.

[16] D. Matignon, "Stability results for fractional differential equations with applications to control processing," IEEE-SMC Computational Engineering in Systems Applications, vol. 2, pp. 963968, 1996.

[17] A. Razminia, V. J. Majd, and D. Baleanu, "Chaotic incommensurate fractional order Rössler system: active control and synchronization," Advances in Difference Equations, vol. 2011, p. 15, 2011.

[18] M. S. Tavazoei, M. Haeri, M. Attari, S. Bolouki, and M. Siami, "More details on analysis of fractional-order Van der Pol oscillator," JVC/Journal of Vibration and Control, vol. 15, no. 6, pp. 803-819, 2009.

[19] M. S. Tavazoei and M. Haeri, "A proof for non existence of periodic solutions in time invariant fractional order systems," Automatica, vol. 45, no. 8, pp. 1886-1890, 2009.

[20] M. S. Tavazoei, "A note on fractional-order derivatives of periodic functions," Automatica, vol. 46, no. 5, pp. 945-948, 2010.

[21] L. O. Chua and M. Itah, "Chaos synchronization in Chua's circuits," Journal of Circuits, Systems and Computers, vol. 3, no. 1, pp. 93-108, 1993.

[22] L. O. Chua, T. Yang, and G. Q. Zhong, "Adaptive synchronization of Chua's oscillator," International Journal of Bifurcation and Chaos, vol. 6, no. 1, pp. 189-201, 1996.

[23] G. R. Chen and X. Dong, From Chaos to Order, World Scientific, Singapore, 1998.

[24] L. M. Pecora and T. L. Carroll, "Synchronization in chaotic systems," Physical Review Letters, vol. 64, no. 8, pp. 821-824, 1990.

[25] X. Wu and H. Wang, "A new chaotic system with fractional order and its projective synchronization," Nonlinear Dynamics, vol. 61, no. 3, pp. 407-417, 2010.

[26] M. P. Aghababa, "Robust stabilization and synchronization of a class of fractional-order chaotic systems via a novel fractional sliding mode controller," Communications in Nonlinear Science and Numerical Simulation, vol. 17, no. 6, pp. 2670-2681, 2012.

[27] M. P. Aghababa, "finite-time chaos control and synchronization of fractional-order nonautonomous chaotic (hyperchaotic) systems using fractional non-singular terminal sliding mode technique," Nonlinear Dynamics, vol. 69, no. 1-2, pp. 247-261, 2012.

[28] J. Kengne, J. C. Chedjou, V. A. Fono, and K. Kyamakya, "On the analysis of bipolar transistor based chaotic circuits: case of a two-stage colpitts oscillator," Nonlinear Dynamics, vol. 67, no. 2, pp. 1247-1260, 2012. 


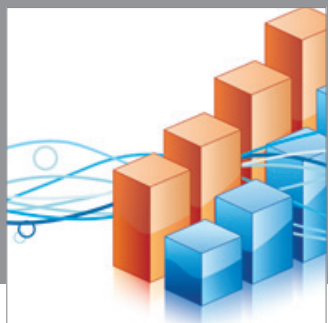

Advances in

Operations Research

mansans

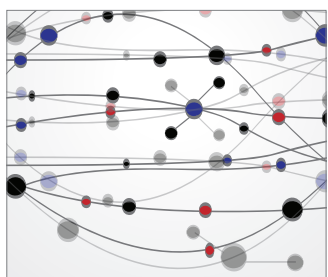

The Scientific World Journal
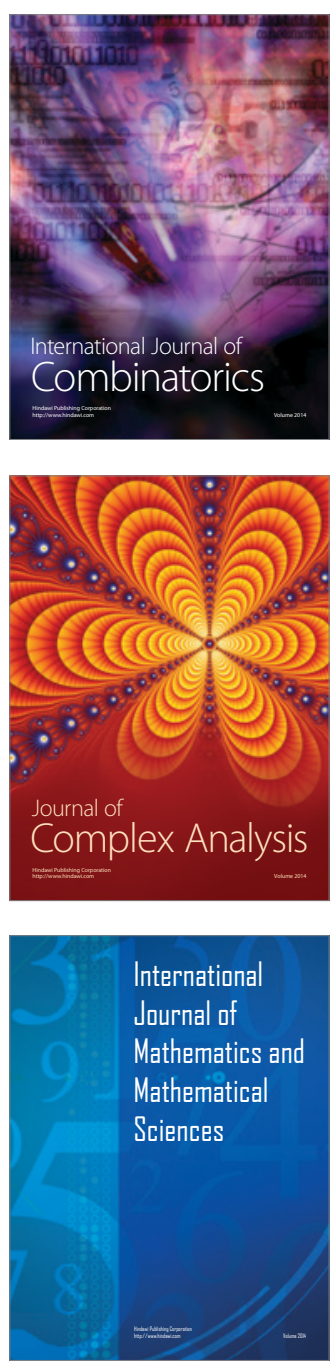
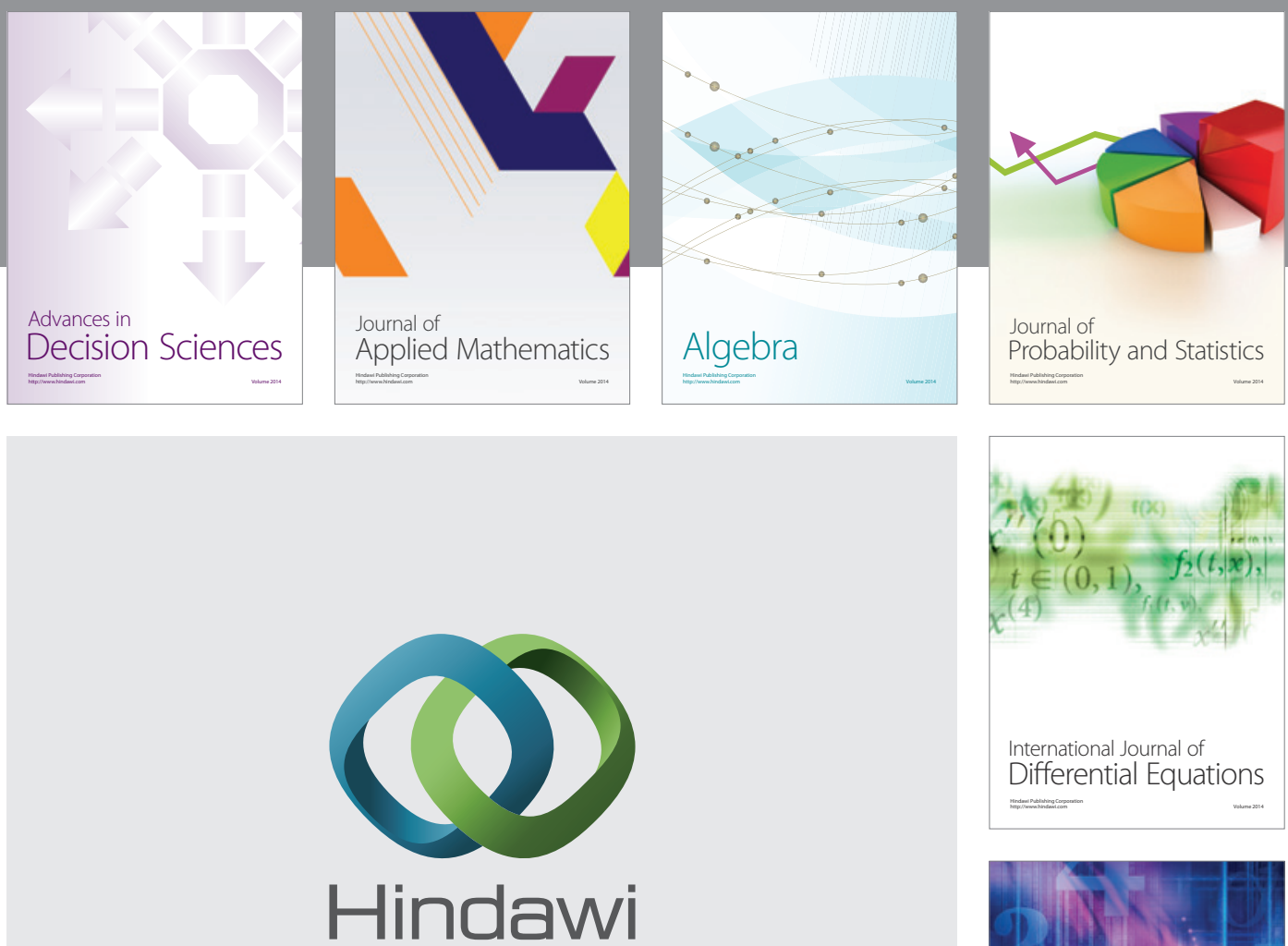

Submit your manuscripts at http://www.hindawi.com
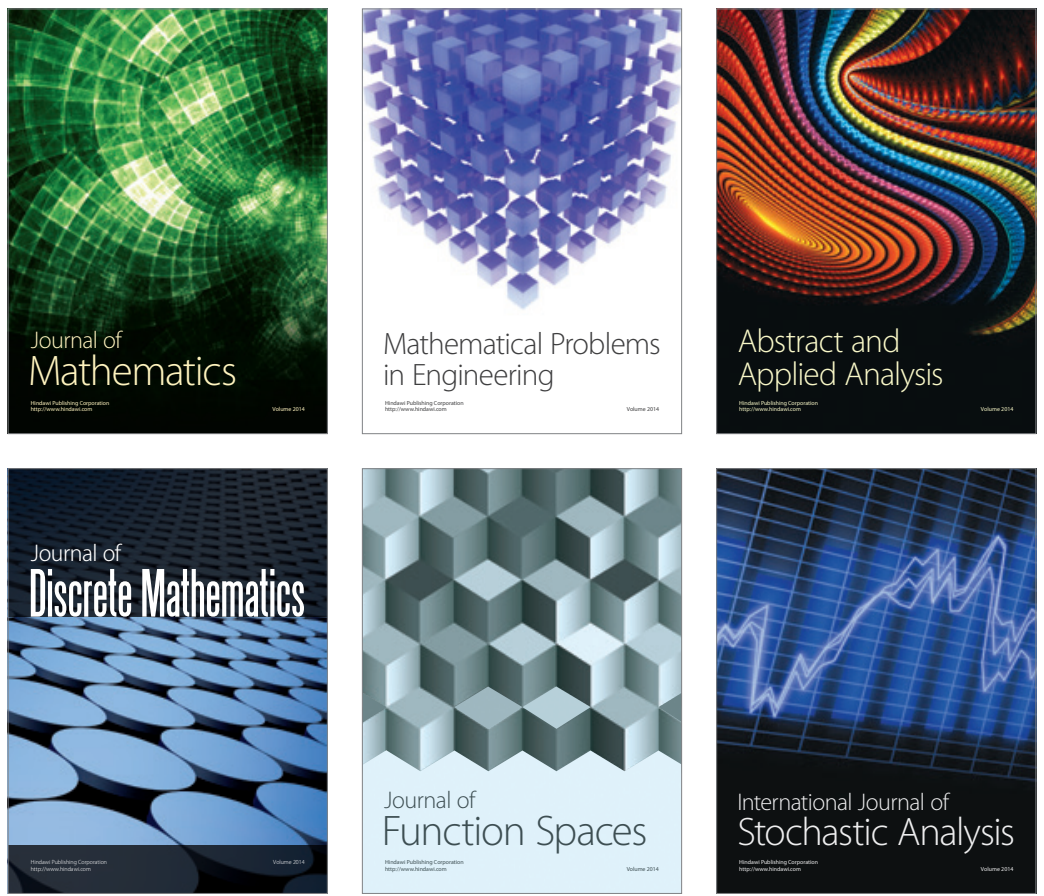

Journal of

Function Spaces

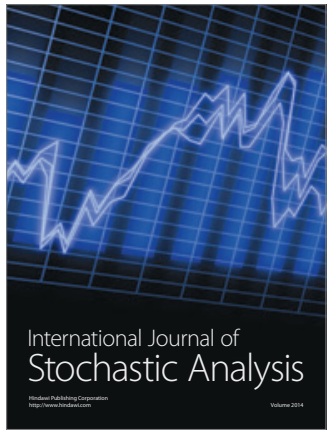

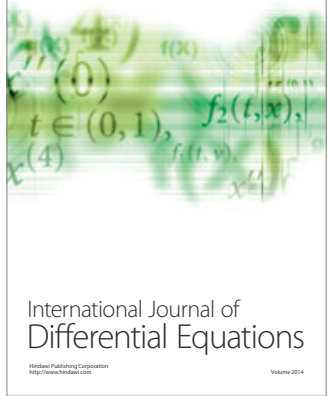
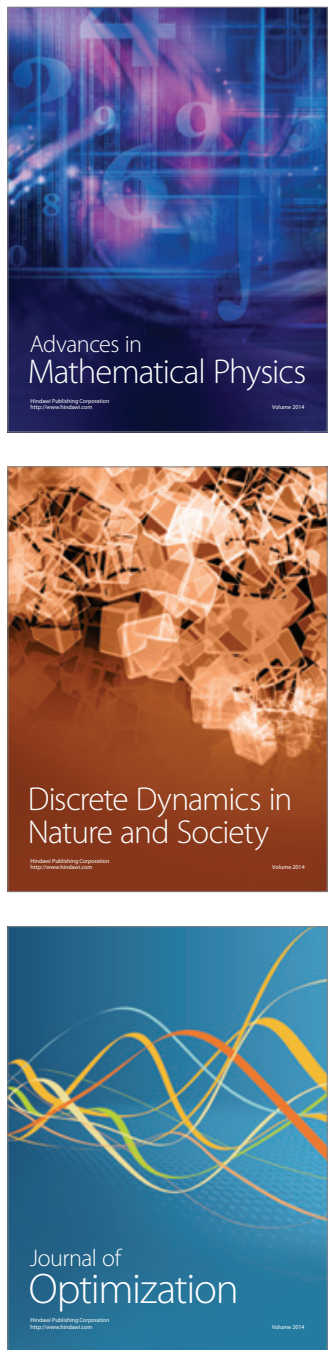